\title{
The impact of chronotropic incompetence on atrioventricular conduction times in heart failure patients
}

\author{
Hongxia Niu ${ }^{1}$, Yinghong $\mathrm{Yu}^{2}$, Vasanth Ravikumar ${ }^{2}$, and Michael Gold ${ }^{3}$ \\ ${ }^{1}$ Chinese Academy of Medical Sciences \& Peking Union Medical College Fuwai Hospital \\ ${ }^{2}$ Boston Scientific Corp Arden Hills \\ ${ }^{3}$ Medical University of South Carolina
}

January 17, 2022

\begin{abstract}
Objectives: To evaluate changes in intrinsic atrioventricular (AV) conduction associated with exercise and atrial pacing among heart failure patients with cardiac resynchronization therapy (CRT). Methods: RAVE was a multicenter prospective trial of CRT patients. Heart rate was increased with incremental atrial pacing up to $130 \mathrm{beats} / \mathrm{min}$ and with submaximal exercise without atrial pacing. According to maximal heart rate achieved during exercise, patients whose maximal heart rate $<100 \mathrm{bpm}$ or $75 \%$ of $85 \%$ of age predicted max heart rate were diagnosed with chronotropic incompentence(CI). Others were classified as patients with chronotropic compentence $(\mathrm{CC})$. For CI patients, an additional symptom limited exercise with rate adaptive pacing activated was performed. Intracardiac intervals were measured from the implantable lead electrograms. Results: There were 12 subjects with CI and 24 with CC. With atrial pacing, AV interval immediately increased and gradually increased with incremental atrial pacing in all patients. However, the changes in the atrial to right ventricular (ARV) and atrial to left ventricular (ALV) intervals with increasing atrial pacing rates were about 3-fold greater in CI patients compared to CC patients ( $28.3 \pm 29.5$ vs. $10.8 \pm 8.9 \mathrm{~ms} / 10 \mathrm{bpm}$ for ARV and $25.5 \pm 22.1$ vs. $9.9 \pm 8.8 \mathrm{~ms} / 10 \mathrm{bpm}$ for ALV in the supine position, p < 0.05$)$. In CI pacing with rate-adaptive pacing during exercise, AV interval changes with paced heart rate were variable. Conclusions: $\mathrm{AV}$ response to overdrive atrial pacing at rest may provide a simple means of identifying chronotropic competence in CRT patients. For patients with CI, who often require rate-adaptive atrial pacing, rate-adaptive AV algorithms should be adjusted individually.
\end{abstract}

\section{INTRODUCTION}

Patients with heart failure with a reduced ejection fraction (HFrEF) have a high incidence of chronotropic incompetence (CI), which is often exacerbated with medical therapy such as beta-adrenergic blockers, antiarrhythmic drugs, or digoxin. ${ }^{1-3}$ As such, rate-adaptive atrial pacing may be beneficial, especially among patients who are physically active. ${ }^{4-5}$ In HFrEF patients with QRS prolongation, cardiac resynchronization therapy (CRT) has been shown to improve exercise tolerance as well as functional status. ${ }^{6-9}$ However, the pivotal CRT trials were performed in the absence of rate-responsive atrial pacing. More recent trials demonstrated the safety of rate-responsive pacing in this population. ${ }^{10}$ However, rate-responsive pacing also affects atrioventricular $(\mathrm{AV})$ conduction times. This can result in suboptimal timing of ventricular pacing, including pseudo fusion or inhibition of pacing. In addtion, AV intervals are used for programming AV delays in several CRT systems. ${ }^{11-13}$ Accordingly, the RAVE study was designed to assess the change in AV conduction with exercise and atrial pacing to help guide the identification of patients with CI, as well as programming of rate responsive AV delay.

\section{METHODS}

RAVE was a multicenter, prospective study of CRT patients in normal sinus rhythm. The study protocol 
was approved by the institutional review board of each center and all patients provided written informed consent.

\subsection{Patients}

Patients were required to be in sinus rhythm with intact AV conduction and be receiving CRT for at least 3 weeks post-device implantation. The exclusion criteria included contraindication to exercise testing. A more detailed description of the study protocol has been published previously. ${ }^{14}$

\subsection{Study protocol}

The RAVE study protocol is shown in Figure 1. First, heart rate (HR) was increased with incremental atrial pacing up to 130 beats/min (bpm) at intervals of $10 \mathrm{bpm}$ in three different postures (supine, standing, and sitting with sitting being optional) to assess the relationship of atrial pacing rate and posture on AV timing (protocol A). Then, a symptom-limited sub-maximal exercise treadmill test was performed in the absence of pacing (protocol B). The ACSM exercise testing and prescription guidelines ${ }^{15}$ were followed to determine chronotropic incompetence. Subsequently, patients who had CI (a maximal heart rate achieved during the submaximal exercise test $<100 \mathrm{bpm}$ or $<63.8 \%$ of age-predicted max heart rate) continued an additional symptom-limited exercise with rate-adaptive atrial pacing with nominal settings activated (protocol C). Of note, only one CI patient had a heart rate $>100 \mathrm{bpm}$. At least 5 minutes was required between each stage of the recordings.

\subsection{Data collection and analysis}

The real-time electrogram data of the implanted CRT device were collected from the right atrial, right ventricular, and left ventricular leads. Atrial to right ventricular (ARV), atrial to the left ventricular (ALV), and RV to LV time intervals were recorded. ARV was defined as the interval between the peaks of RA and RV electrograms, and ALV interval was the interval between the peaks of RA and LV electrograms. Ectopic beats, noisy signals, and the intervals due to under-sensing or over-sensing were removed prior to analysis.

All data analyses used MATLAB (Natick, MA) and SAS (Cray, NC) software. Continuous variables were expressed as mean \pm standard deviation or median (interquartile range (IQR)), where appropriate. A linear regression line was fitted between AV intervals and heart rates, and the slope of the line was reported to assess the relationship between AV intervals and exercise/pacing. An unpaired t-test was performed for significance testing, and a p-value of [?]0.05 was considered statistically significant. For categorical variables, a Fisher exact test was performed.

\section{RESULTS}

\subsection{Baseline characteristics}

There were 36 heart failure patients enrolled in the RAVE study. According to the maximal heart rate achieved during the symptom-limited exercise test, patients were divided into 2 groups: Group 1: CI patients $(n=12)$; and group 2: chronotropic competent $(\mathrm{CC})$ patients $(\mathrm{n}=24)$. The baseline characteristics of the groups are shown in Table 1. This was a typical cohort of heart failure patients and the groups were well matched, except that CI patients had a higher ejection fraction than CC subjects $(29+-5 \%$ vs. $23+-$ $6 \%, \mathrm{p}<0.05)$. Based on group classification, compared to CC patients, CI patients had significantly lower maximum heart rate during the submaximal exercise test in the absence of pacing (protocol B) (86.7 +11.8bpm Vs. $110.7+-25.0 \mathrm{bpm}, \mathrm{p}<0.001)$.

\subsection{AV interval changes in CI and CC patients at rest}

During protocol A, incremental atrial pacing at different postures was performed to study the changes in AV intervals with respect to HR. Both ARV and ALV increased with incremental atrial pacing compared to intrinsic intervals. As shown previously, ARV and ALV intervals increased rapidly in all subjects from atrial sense to atrial pacing as shown by a larger slope and then increased gradually as the paced heart rate increased, as shown by a smaller slope. ${ }^{14}$ New data reported here shows that patients with CI have 
significantly greater prolongation of AV intervals than CC patients. These results are summarized in Table 2. The averaged slopes of prolongation of ARV and ALV intervals with increasing paced heart rate for CI patients were $>20 \mathrm{~ms}$ per 10 beat increase in atrial pacing rates for all postures. In contrast, in CC patients, the averaged slopes of prolongation of AV intervals with paced rates were almost always $<10 \mathrm{~ms}$ per 10 beat increase in pacing rates for each posture. The changes in ARV and ALV were similar for all postures in both groups $(\mathrm{p}=\mathrm{NS})$. Overall, the prolongation of AV intervals was about 3-fold greater in patients with CI compared with CC for all postures $(\mathrm{p}<0.05)$.

\subsection{AV interval changes in CI patients during exercise testing}

In the CI patients, submaximal exercise testing was performed first (protocol B) with no atrial pacing. Next, rate-adaptive atrial pacing was activated (protocol $\mathrm{C}$ ). As expected, the maximum heart rate during exercise was higher when the rate-adaptive pacing was present, as shown in Figure 2. ARV and ALV slopes during standing at rest, exercise with no pacing, and exercise with rate-adaptive atrial pacing activated are summarized in Table 3. It was observed that the slope of prolongation of AV intervals vs. atrial paced heart rate (protocol C) was larger with rate-adaptive pacing turned on as compared to the slope of prolongation of AV intervals vs. atrial sensed heart rate during exercise (protocol B) in CI patients (Figure 3).

During the exercise test for CI patients in protocol C, except for one patient, there was initially rapid increase in $\mathrm{AV}$ intervals with the transition from atrial sensing (As) to atrial pacing (Ap) (ARV slope $=57.4+-64.8$ $\mathrm{ms} / 10 \mathrm{bpm}$ and ALV slope $=68.7+-76.9 \mathrm{~ms} / 10 \mathrm{bpm}$ ). This was followed by continuous but more gradual increases in AV intervals as heart rate increased (ARV slope $=14.0+-12.9$ and ALV slope $=13.6+-13.8$ $\mathrm{ms} / 10 \mathrm{bpm}$ ). The trends were similar to those with atrial pacing at rest. However, the changes in AV intervals were variable among different CI patients. Only one patient showed a decreased AV interval from As to Ap. Specifically, 6 patients had a slope of ARV vs. heart rate $>5 \mathrm{~ms} / 10 \mathrm{bpm}$, and 4 patients had a slope of ALV vs. heart rate $>5 \mathrm{~ms} / 10 \mathrm{bpm}$ during increased $\mathrm{HR}$ with rate-adaptive pacing activated.

\section{DISCUSSION}

\subsection{Main findings}

RAVE is the first multicenter trial to evaluate the impact of posture, atrial pacing, and exercise on inter and intraventricular delays in CRT patients. Such delays are important to maintain a high percentage of ventricular pacing, but are also used to optimize fusion with intrinsic conduction. We report 4 major findings from this study. First, the changes in AV conduction are very different in CI and CC patients with atrial pacing. Second, posture has little effect on AV conduction, so device AV delay programming and optimization algorithms do not need to adjust for posture. Third, a sudden and large increase in AV conduction is observed with atrial pacing, which should help guide the programming of AV delays during such pacing.Finally, the change in AV conduction with exercise in patients with CI is variable, so individualization is likely needed rather than using nominal settings.

About $30 \%$ of all CRT patients are classified as nonresponders, although the classification system has recently been questioned. ${ }^{16}$ Nevertheless, a weaker response to CRT may be partly explained by the inappropriate programming of $\mathrm{AV}$ intervals. ${ }^{17}$ Although guidelines do not recommend routine optimization, ${ }^{4,18}$ current electrogram algorithms ${ }^{11-13}$ are frequently based on achieving fusion with intrinsic conduction, so such intervals are important.

\subsection{Differeent changes in AV interval with atrial pacing in $\mathrm{CI}$ and $\mathrm{CC}$ patients}

In this study, one-third of patients were diagnosed with CI, and overdriving atrial pacing markedly increased the AV interval in those patients. The prolongation of AV intervals was about 3-fold greater in patients with CI compared with CC. This result has important implications, as it may provide a simple method to identify CI patients according to the response to overdriving atrial pacing without the need for exercise testing. In clinical practice, CI is frequently underdiagnosed as formal exercise testing is not performed routinely. CI is a major limiting factor for exercise capacity, and it is also a predictor of cardiovascular events and mortality. ${ }^{1,5,19,20}$ Consequently, timely and easily identified CI patients will allow activation of 
rate-adaptive pacing, which can improve patients' exercise tolerance, functional class, quality of life, and potentially improve prognosis. ${ }^{21,22}$

A second observation from this study is that high-rate atrial pacing at rest caused the significant lengthening of intrinsic AV interval. Notably, atrial pacing rates were increased during rest, which is in the absence of metabolic demand. However, higher basal heart rates are often favored among patients with severe cardiac dysfunction or worsening heart failure. Given a low stroke volume, a higher basic pacing rate even at rest is often needed to maintain cardiac output. For these patients, AV delays should be programmed markedly longer than the nominal settings. Presumably, this slowed AV conduction is due to properties of the AV node as well as possible slowing of conduction through the myocardium in myopathic hearts.

\subsection{Rate adaptive atrial pacing variably lengthened the AV intervals}

CRT AV delay programming is complex due to uncertainty of the optimal AV delay, atrial-paced/sensed offset, and rate-adaptive AV delay. In this study, the intrinsic AV interval with activated rate-adaptive pacing changed individually but on average lengthened. This observation has important implications for dynamic AV delay programming in CRT patients, especially in those who need atrial pacing. The paced AV offset and rate-adaptive changes should be patient-specific and differ significantly from nominal device settings. Automated assessment of AV conduction with atrial pacing may facilitate this response.

Previous studies have shown that the AV delay offset for atrial paced compared with atrial sensed in CRT is often underestimated at normal settings derived from bradycardia pacing patients. ${ }^{23-25}$ Our study indicates a longer paced AV offset may be useful for CRT than most nominal settings. These results provide further support for the physiologic differences in AV conduction in systolic heart failure patients compared with patients with normal systolic function. ${ }^{26}$

For rate-adaptive pacing, progressive shortening of AV interval has been proved to benefit diastolic filling and ejection time, and so empirically programmed in a dual-chamber pacemaker. However, recent studies have suggested that this algorithm does not work well for biventricular pacing. ${ }^{27-29}$ Due to the uncertainty and inconsistency in this aspect, the AV delay is often programmed to be fixed in most modern biventricular devices. However, our results suggest that lengthening of the rate-adaptive AV delay may be more suitable for heart failure patients, which differs significantly from the traditional device settings.

\section{LIMITATIONS}

This study should be interpreted in light of several methodologic limitations. First, the number of patients studied was relatively small. Second, the data were collected over a relatively short period of time, so the impact of prolonged pacing or exercise was not assessed. Finally, further study is needed to develop algorithms for AV delays in sensor-driven as well as programmed changes in heart rate.

\section{CONCLUSIONS}

The AV response to overdrive atrial pacing at rest may provide a simple means of identifying chronotropic competence in CRT patients. For patients with CI, who often require rate-adaptive atrial pacing, the intrinsic AV interval changed individually but on average lengthened. Accordingly, optimization of AV delay at rest is not suitable for all activity levels. Rate Adaptive AV should be individually determined and dynamically optimized according to the increased heart rate.

\section{ACKNOWLEDGMENT: None.}

\section{ORCID}

Hongxia Niu https://orcid.org/0000-0003-3033-4741

Yinghong Yu https://orcid.org/0000-0002-6314-8390

Vasanth Ravikumar https://orcid.org/0000-0002-8959-3214

Michael R. Gold https://orcid.org/0000-0002-4579-0216 


\section{REFERENCES}

1. Al-Najjar Y, Witte KK, Clark AL. Chronotropic incompetence and survival in chronic heart failure. Int J Cardiol.2012;157:48-52.

2. Jorde UP, Vittorio TJ, Kasper ME, et al. Chronotropic incompetence, beta-blockers, and functional capacity in advanced congestive heart failure: time to pace? Eur J Heart Fail. 2008;10:96-101.

3. Brubaker PH, Kitzman DW. Prevalence and management of chronotropic incompetence in heart failure. Curr Cardiol Rep.2007;9:229-235.

4. Kusumoto FM, Schoenfeld MH, Barrett C, et al. 2018 ACC/AHA/HRS Guideline on the Evaluation and Management of Patients With Bradycardia and Cardiac Conduction Delay: A Report of the American College of Cardiology/American Heart Association Task Force on Clinical Practice Guidelines and the Heart Rhythm Society. Circulation. 2019;140:e382-e482.

5. Brubaker PH, Kitzman DW. Chronotropic incompetence: causes, consequences, and management. Circulation. 2011;123:1010-1020.

6. Abraham WT, Fisher WG, Smith AL, et al. MIRACLE Study Group. Multicenter InSync Randomized Clinical Evaluation. Cardiac resynchronization in chronic heart failure. N Engl J Med.2002;346:18451853.

7. Bristow MR, Saxon LA, Boehmer J, et al. Comparison of Medical Therapy, Pacing, and Defibrillation in Heart Failure (COMPANION) Investigators. Cardiac-resynchronization therapy with or without an implantable defibrillator in advanced chronic heart failure. $N$ Engl $J$ Med.2004;350:2140-2150.

8. Linde C, Abraham WT, Gold MR, et al; REVERSE (REsynchronization reVErses Remodeling in Systolic left vEntricular dysfunction) Study Group. Randomized trial of cardiac resynchronization in mildly symptomatic heart failure patients and in asymptomatic patients with left ventricular dysfunction and previous heart failure symptoms.J Am Coll Cardiol. 2008;52:1834-1843.

9. Moss AJ, Hall WJ, Cannom DS, et al; MADIT-CRT Trial Investigators. Cardiac-resynchronization therapy for the prevention of heart-failure events. N Engl J Med. 2009;361:1329-1338.

10. Martin DO, Day JD, Lai PY, et al. Atrial support pacing in heart failure: results from the multicenter PEGASUS CRT trial. J Cardiovasc Electrophysiol. 2012;23:1317-1325.

11. Ellenbogen KA, Gold MR, Meyer TE, et al. Primary results from the SmartDelay determined AV optimization: a comparison to other AV delay methods used in cardiac resynchronization therapy (SMART-AV) trial: a randomized trial comparing empirical, echocardiography-guided, and algorithmic atrioventricular delay programming in cardiac resynchronization therapy. Circulation. 2010;122:26602668.

12. Martin DO, Lemke B, Birnie D, et al; Adaptive CRT Study Investigators. Investigation of a novel algorithm for synchronized left-ventricular pacing and ambulatory optimization of cardiac resynchronization therapy: results of the adaptive CRT trial. Heart Rhythm.2012;9:1807-1814.

13. Ter Horst IAH, Bogaard MD, Tuinenburg AE,et al. The concept of triple wavefront fusion during biventricular pacing: Using the EGM to produce the best acute hemodynamic improvement in CRT. Pacing Clin Electrophysiol. 2017;40:873-882.

14. Niu H, Yu Y, Sturdivant JL, An Q, Gold MR. The effect of posture, exercise, and atrial pacing on atrioventricular conduction in systolic heart failure. J Cardiovasc Electrophysiol. 2019;30:2892-2899.

15. Armstrong Lawrence E., Brubaker Peter H., Otto Robert M. ACSM's Guidelines for Exercise Testing and Prescription. 7th ed. Baltimore: Lippincott Williams \& Wilkins; 2006.

16. Gold MR, Rickard J, Daubert JC, Zimmerman P, Linde C. Redefining the Classifications of Response to Cardiac Resynchronization Therapy: Results From the REVERSE Study. JACC Clin Electrophysiol . 2021;7:871-880.

17. Mullens W, Grimm RA, Verga T, et al. Insights from a cardiac resynchronization optimization clinic as part of a heart failure disease management program. J Am Coll Cardiol . 2009;53:765-773.

18. Daubert JC, Saxon L, Adamson PB, et al. 2012 EHRA/HRS expert consensus statement on cardiac resynchronization therapy in heart failure: implant and follow-up recommendations and management. Heart Rhythm . 2012;9:1524-1576.

19. Lauer MS, Okin PM, Larson MG, Evans JC, Levy D. Impaired heart rate response to graded exercise. 
Prognostic implications of chronotropic incompetence in the Framingham Heart Study. Circulation . 1996;93:1520-1526.

20. Zweerink A, van der Lingen ACJ, Handoko ML, van Rossum AC, Allaart CP. Chronotropic Incompetence in Chronic Heart Failure. Circ Heart Fail . 2018;11:e004969.

21. Palmisano P, Aspromonte V, Ammendola E, et al; Italian Association of Arrhythmology and Cardiac Pacing (AIAC). Effect of fixed-rate vs. rate-RESPONSIve pacing on exercise capacity in patients with permanent, refractory atrial fibrillation and left ventricular dysfunction treated with atrioventricular junction aBLation and bivEntricular pacing (RESPONSIBLE): a prospective, multicentre, randomized, single-blind study. Europace. 2017;19:414-420.

22. Olshansky B, Richards M, Sharma A, et al. Survival After Rate-Responsive Programming in Patients With Cardiac Resynchronization Therapy-Defibrillator Implants Is Associated With a Novel Parameter: The Heart Rate Score. Circ Arrhythm Electrophysiol . 2016;9:e003806.

23. Riedlbauchova L, Kautzner J, Fridl P. Influence of different atrioventricular and interventricular delays on cardiac output during cardiac resynchronization therapy. Pacing Clin Electrophysiol . 2005;28 Suppl 1:S19-23.

24. Tse HF, Siu CW, Lee KL, et al. The incremental benefit of rate-adaptive pacing on exercise performance during cardiac resynchronization therapy. J Am Coll Cardiol . 2005;46:2292-2297.

25. Di Pede F, Gasparini G, De Piccoli B, Yu Y, Cuesta F, Raviele A. Hemodynamic effects of atrial septal pacing in cardiac resynchronization therapy patients. J Cardiovasc Electrophysiol 2005;16:1273-1278.

26. Gold MR, Niazi I, Giudici M, et al. Acute hemodynamic effects of atrial pacing with cardiac resynchronization therapy. J Cardiovasc Electrophysiol 2009;20:894-900.

27. Bogaard MD, Kirkels JH, Hauer RN, Loh P, Doevendans PA, Meine M. Should we optimize cardiac resynchronization therapy during exercise? J Cardiovasc Electrophysiol 2010;21:1307-1316.

28. Shanmugam N, Prada-Delgado O, Campos AG, et al. Rate-adaptive AV delay and exercise performance following cardiac resynchronization therapy. Heart Rhythm 2012;9:1815-1821.

29. Thibault B, Ritter P, Bode K, Calo L, et al. Dynamic programming of atrioventricular delay improves electrical synchrony in a multicenter cardiac resynchronization therapy study. Heart Rhythm 2019;16:1047-1056.

Table 1. Baseline demographics for CI and CC patients. CI, chronotropic incompetence; CC, chronotropic competence; LBBB, left bundle branch block.

\begin{tabular}{llll}
\hline & CI pts (n=12) & CC pts (n=24) & P value \\
\hline Age (years) & $67 \pm 9$ & $64 \pm 10$ & 0.37 \\
Male (\%) & 75 & 71 & 0.56 \\
Ischemic Cardiomyopathy (\%) & 83 & 46 & 0.034 \\
NYHA functional class III (\%) & 50 & 46 & 0.55 \\
NYHA functional class II (\%) & 42 & 36 & 0.45 \\
NYHA functional class I (\%) & 8 & 18 & 0.89 \\
Left-ventricular ejection fraction (\%) & $29 \pm 5$ & $23 \pm 6$ & 0.025 \\
LBBB (\%) & 67 & 83 & 0.88 \\
QRS duration (ms) & $165 \pm 27$ & $170 \pm 30$ & 0.611 \\
PR interval (ms) & $191 \pm 40$ & $186 \pm 33$ & 0.715 \\
Maximal Heart Rate during 1st exercise test (bpm) & $87 \pm 12$ & $111 \pm 25$ & 0.001 \\
Beta blocker (\%) & 92 & 100 & 1 \\
Digoxin (\%) & 25 & 17 & 0.43 \\
\hline
\end{tabular}

Table 2 The rates of change of ARV and ALV intervals with increasing paced atrial rates. ${ }^{*} \mathrm{p}<0.05$ compared with CC. Unit: ms/10 bpm. ARV, atrial to right ventricular; ALV, atrial to the left ventricular; CC, chronotropic competence; CI, chronotropic incompetence 


\begin{tabular}{lllllll}
\hline & Supine & Supine & Standing & Standing & Sitting & Sitting \\
\hline & ARV slope* & ALV slope* & ARV slope* & ALV slope* & ARV slope & ALV slope \\
CC patients & $10.81 \pm 8.92$ & $9.85 \pm 8.75$ & $7.18 \pm 5.51$ & $7.09 \pm 5.68$ & $8.81 \pm 5.35$ & $8.51 \pm 6.15$ \\
CI patients & $28.32 \pm 29.51$ & $25.45 \pm 22.07$ & $24.29 \pm 28.90$ & $22.73 \pm 25.56$ & $20.14 \pm 24.01$ & $20.75 \pm 24.75$ \\
\hline
\end{tabular}

Table $3 \mathrm{ARV}$ and ALV slopes during standing at rest (protocol A), exercise with rate-adaptive atrial pacing activated (Protocol C), and exercise with no pacing (Protocol B) in chronotropic incompetence patients. Unit: ms/10 bpm. ARV, atrial to right ventricular; ALV, atrial to left ventricular; As, atrial sensing; Ap, atrial pacing; HR, heart rate.

\begin{tabular}{lllllll}
\hline & ARV slope & ARV slope & ARV slope & ARV slope & ARV slope & $A L V$ \\
\hline & Protocol A & Protocol A & Protocol C & Protocol C & Protocol B & Protc \\
& From As to Ap & Ap with increased HR & From As to Ap & Ap with increased HR & Exercise with no pacing & From \\
1 & 136.1 & 10.9 & 49.4 & 6.0 & 23.2 & 128.6 \\
2 & - & 108.2 & 198.1 & - & 42.7 & - \\
3 & -17.7 & 36.6 & -1.4 & 2.9 & 4.78 & 55.3 \\
4 & 23.4 & 14.5 & 38.3 & 5.7 & -4.11 & 26.6 \\
5 & - & 28.1 & - & 31.2 & 4.63 & - \\
6 & - & 14.3 & -5.8 & 2.5 & 0.16 & 179.3 \\
7 & 176.0 & 38.9 & 50.3 & 32.7 & -8.98 & 205.0 \\
8 & 206.4 & 14.3 & - & 12.2 & 4.34 & 38.3 \\
9 & 38 & 5.3 & 21.5 & 28.3 & -2.57 & 48.9 \\
10 & 48.9 & 7.9 & $57.4 \pm 64.8$ & $14.0 \pm 12.9$ & -0.85 & $97.4 \pm$ \\
avg & $87.3 \pm 85.1$ & $27.9 \pm 30.6$ & & & & $6.3 \pm 16.0$ \\
\hline
\end{tabular}

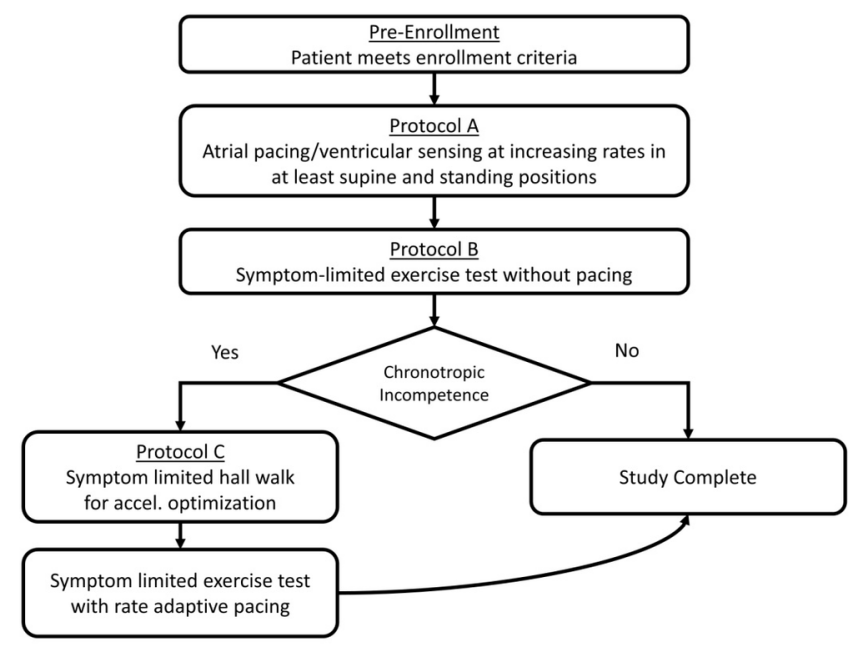

Figure 1. RAVE study protocol flow. 


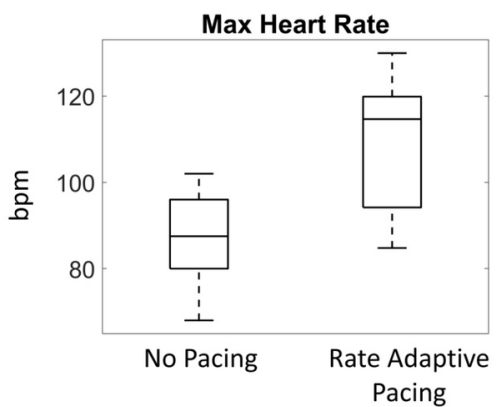

Figure 2 Maximum heart rate in chronotropic incompetence patients during exercise tests with no atrial pacing (protocol B) and rate-adaptive atrial pacing (protocol C).
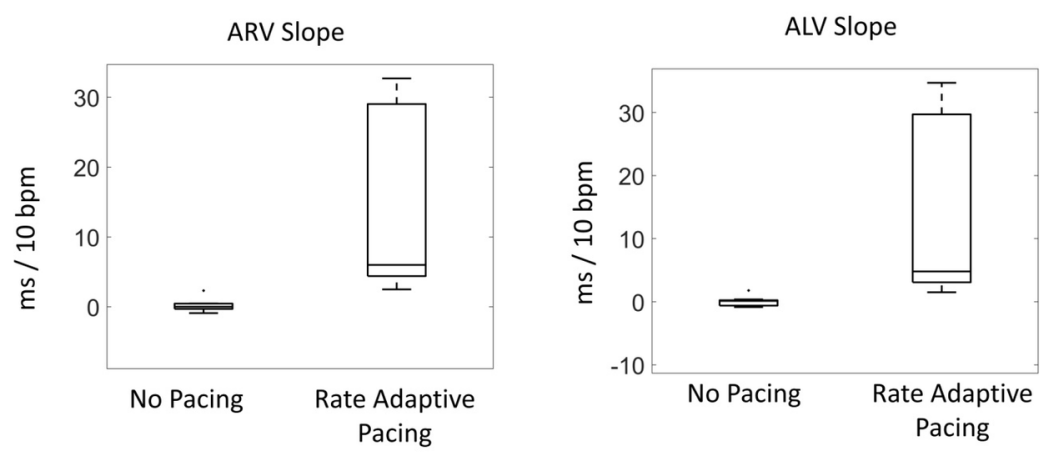

Figure $3 \mathrm{ARV}$ and ALV slope in chronotropic incompetence patients during exercise with no atrial pacing (protocol B) and rate-adaptive atrial pacing (protocol C). ARV, Atrial to right ventricular; ALV, atrial to left ventricular. 